\title{
Posterior thoracic laminoplasty with dorsal, intradural identification of ventral defect and transdural discectomy for a spontaneous cerebrospinal fluid leak: case report
}

\author{
Katie Pricola Fehnel, MD, 1,2 and Lawrence F. Borges, MD'1 \\ 'Department of Neurosurgery, Massachusetts General Hospital; and 2Vascular Biology Program and Department of \\ Neurosurgery, Boston Children's Hospital, Boston, Massachusetts
}

\begin{abstract}
Spontaneous intracranial hypotension $(\mathrm{SIH})$ has been increasingly reported in the literature concomitant with the improved sensitivity of imaging modalities. Although typically associated with meningeal weakening, a handful of cases of SIH secondary to thoracic disc osteophytes have been reported. Five of 7 reported cases were treated with epidural blood patch (EBP) alone while 2 required surgical management. There is no standard operative approach; both anterior and posterolateral approaches can be cumbersome and associated with morbidity, particularly in young, healthy patients. The authors report a case of SIH in which a ventral dural tear secondary to a calcified thoracic disc was repaired via posterior thoracic laminoplasty with dorsal durotomy and intradural exposure of the ventral defect with transdural discectomy followed by primary closure.
\end{abstract}

A 34-year-old man presented with low-pressure headaches following axial load injury from a ski accident 5 years earlier. The patient's symptoms were refractory to a trial of conservative treatment and EBP, and he developed bilateral upper-extremity paresthesias. MRI of the spine demonstrated an extrathecal collection spanning the thoracic spine, and dynamic CT myelography identified contrast extravasation adjacent to a calcified paramedian disc at T9-10. The patient underwent posterior laminoplasty with neuromonitoring. A ventral dural defect was visualized via a dorsal durotomy, the penetrating disc osteophyte was removed transdurally, and the ventral and dorsal dura maters were closed primarily. Both somatosensory and motor evoked potentials were unchanged during surgery. The patient has remained asymptomatic more than 10 months postoperatively and he has resumed work as a surgeon.

Cases of SIH secondary to a calcified thoracic disc are rare with little precedent as to optimal surgical intervention. This case illustrates the potential usefulness of posterior laminectomy in nonmyelopathic patients in whom there is no evidence of canal compromise and for whom neuromonitoring is available. Additionally, surgeon experience and patient preference may guide surgical planning.

http://thejns.org/doi/abs/10.3171/2014.10.SPINE14439

KEY WORDS spontaneous intracranial hypotension; thoracic laminectomy; transdural discectomy

$\mathrm{S}$ PONTANEOUS intracranial hypotension $(\mathrm{SIH})$ has been increasingly reported in the literature as imaging modalities have improved. Magnetic resonance imaging can detect associated intracranial findings, such as enhancing meninges, tonsillar descent, dural sinus engorgement, and pituitary enlargement. ${ }^{7,13,14,17,21,30}$ The most common etiologies of SIH are arachnoid cysts, torn nerve root sleeves, meningeal diverticula, ${ }^{21,22}$ and, rarely, disc osteophytes. ${ }^{25}$ Only a handful of thoracic disc herniations associated with SIH have been reported in the literature, and, given the paucity of such reports, no consistent approach to surgical intervention has prevailed. We report a case in which a ventral dural tear was identified in a delayed fashion secondary to a calcified thoracic disc. Repair was performed via a posterior thoracic laminoplasty with dorsal durotomy and intradural exposure of the ventral defect with transdural discectomy followed by primary closure. We review the literature with regard to treatment of thoracic disc-associated spontaneous CSF leaks as well as the literature on transdural discectomy.

ABBREVIATIONS EBP = epidural blood patch; MEP = motor evoked potential; $\mathrm{SIH}=$ spontaneous intracranial hypotension; SSEP = somatosensory evoked potential. SUBMITTED June 16, 2014. ACCEPTED October 7, 2014 


\section{Case Report}

History and Presentation

A 34-year-old man presented after 2 months of postural headaches and bilateral shoulder discomfort following axial load injury from a ski accident that occurred 5 years earlier. As a physician, the patient suspected SIH based on his symptoms and had instituted a trial of caffeine, liquids, and ibuprofen with some relief. Initial brain MRI findings were unremarkable, and the patient was sent home from the emergency department. He returned 2 days later with new onset nausea, vomiting, and worsening postural headache. At that time, examination findings remained normal without focal deficit, and findings on noncontrast CT scanning of the head and MRI of the brain with contrast were again unremarkable (Fig. 1A). Spine MRI revealed a 2-mm extrathecal fluid collection spanning from the midthoracic region to the upper lumbar spine (Fig. 1B and $\mathrm{C}$ ), and the patient was admitted for conservative management of a suspected CSF leak. His symptoms persisted after administration of intravenous fluids and analgesia, and ultimately an epidural blood patch (EBP) was performed at L4-5 with $25 \mathrm{ml}$ of autologous peripheral blood. The patient experienced only partial symptom relief and required a second EBP 2 weeks later. Unfortunately, his symptoms acutely worsened following the second EBP, and the neurosurgery service was consulted for possible surgical management; however, the patient did not wish to pursue surgery at that time. After an additional week of persistent symptoms, he returned to the emergency department seeking definitive treatment. His imaging studies and course thereafter are detailed below.
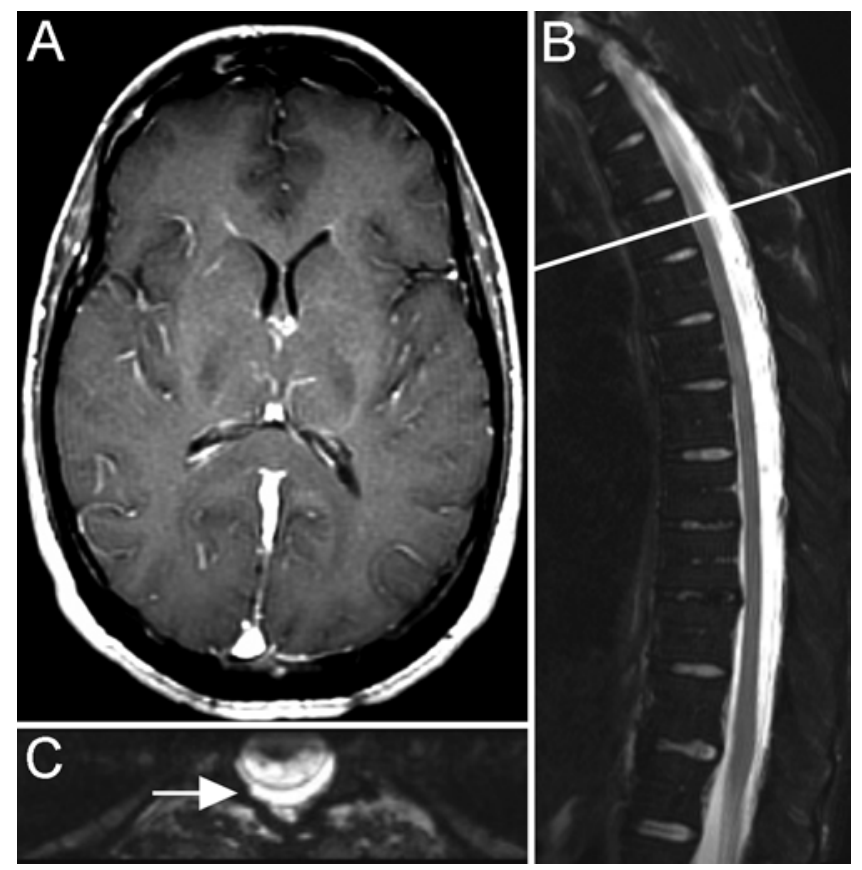

FIG. 1. A: Brain MR image with contrast showing normal findings and no evidence of meningeal enhancement. B and C: Sagittal T2-weighted MR image (B) and axial T2-weighted constructive interference in steady state (CISS) image $(\mathrm{C})$ showing a posterior thoracic extrathecal collection measuring $2 \mathrm{~mm}$ in greatest thickness (arrow). The line in panel $\mathrm{B}$ indicates the axial level in panel $\mathrm{C}$.
Imaging

MRI of the cervical, lumbar, and thoracic spine showed an expanding extradural collection from C-7 to L-1 now measuring $5 \mathrm{~mm}$ in greatest thickness (Fig. 2A and B). A dynamic CT myelogram was obtained as per recent guidelines from the Mayo Clinic, which state that symptomatic patients with extraarachnoid spinal fluid on MRI who have failed conservative management and nontargeted therapy should directly undergo dynamic CT myelography. ${ }^{10}$ Dynamic CT myelography of the cervical, thoracic, and lumbar spine was performed with the patient in the left lateral decubitus position; a scan was obtained after the first 15 $\mathrm{ml}$ of contrast medium was injected at L3-4, and another scan was obtained after injection of an additional $15 \mathrm{ml}$ of contrast medium. Subsequently, 3 more 15-second scans were obtained in the right lateral decubitus, supine, and prone positions. Radiologists reviewed the 5-mm slice images generated from the numerous sequences and selected the highest yield areas through which additional thin-slice axial reformatted images were taken. ${ }^{9}$ CT myelography identified contrast extravasation adjacent to a 2-mm calcified paramedian disc at T9-10 (Fig. 2C and D).

\section{Operation}

The patient was taken to the operating room for thoracic exploration for repair of suspected ventral durotomy and associated disc osteophytectomy. The patient was positioned prone on a Jackson table, with continuous monitoring of motor evoked potentials (MEPs) and somatosensory evoked potentials (SSEPs). Spinal localization was performed prior to incision using 3 sequential needles and a lateral plain radiograph with excellent visualization of the T9-10 disc space. Localization was repeated at the subfascial juncture of the dissection with a second lateral radiograph. A T9-10 laminectomy was then performed using a 1.7-mm diamond matchstick. Dural inspection revealed dorsal scarring, presumably from the prior 2 EBPs while the ventral dura showed no evidence of scarring and obvious CSF extravasation. A dorsal durotomy was performed through a small incision elongated rostral to caudal using a dental instrument. With the dura tented open using 4-0 Nurolon suture, a midline ventral dural defect was identified that measured approximately $5 \mathrm{~mm}$ in length. A small, calcified disc was noted to be penetrating through the dural tear (Fig. 3A) and was resected transdurally through the defect (Fig. 3B). An extremely conservative discectomy was performed, and the annulus was cauterized. MEPs and SSEPs remained baseline throughout. Watertight closure of the ventral defect was performed using a running 5-0 silk suture on a small needle (Fig. 3C). A small autologous fat graft was placed ventral to the durotomy and posterior to the disc space, and watertight closure of the dorsal durotomy was achieved with a running 5-0 Prolene suture and covered with a small autologous fat graft. The T-9 and T-10 laminae were replaced with a 2.0 titanium miniplate (Synthes), and the remainder of the closure was performed in the standard fashion. MEPs improved from baseline to conclusion of the procedure (180 and $190 \mathrm{~mA}$ on the right and left sides, respectively, from 160 and 170 $\mathrm{mA}$ preoperatively), and SSEPs remained unchanged. 

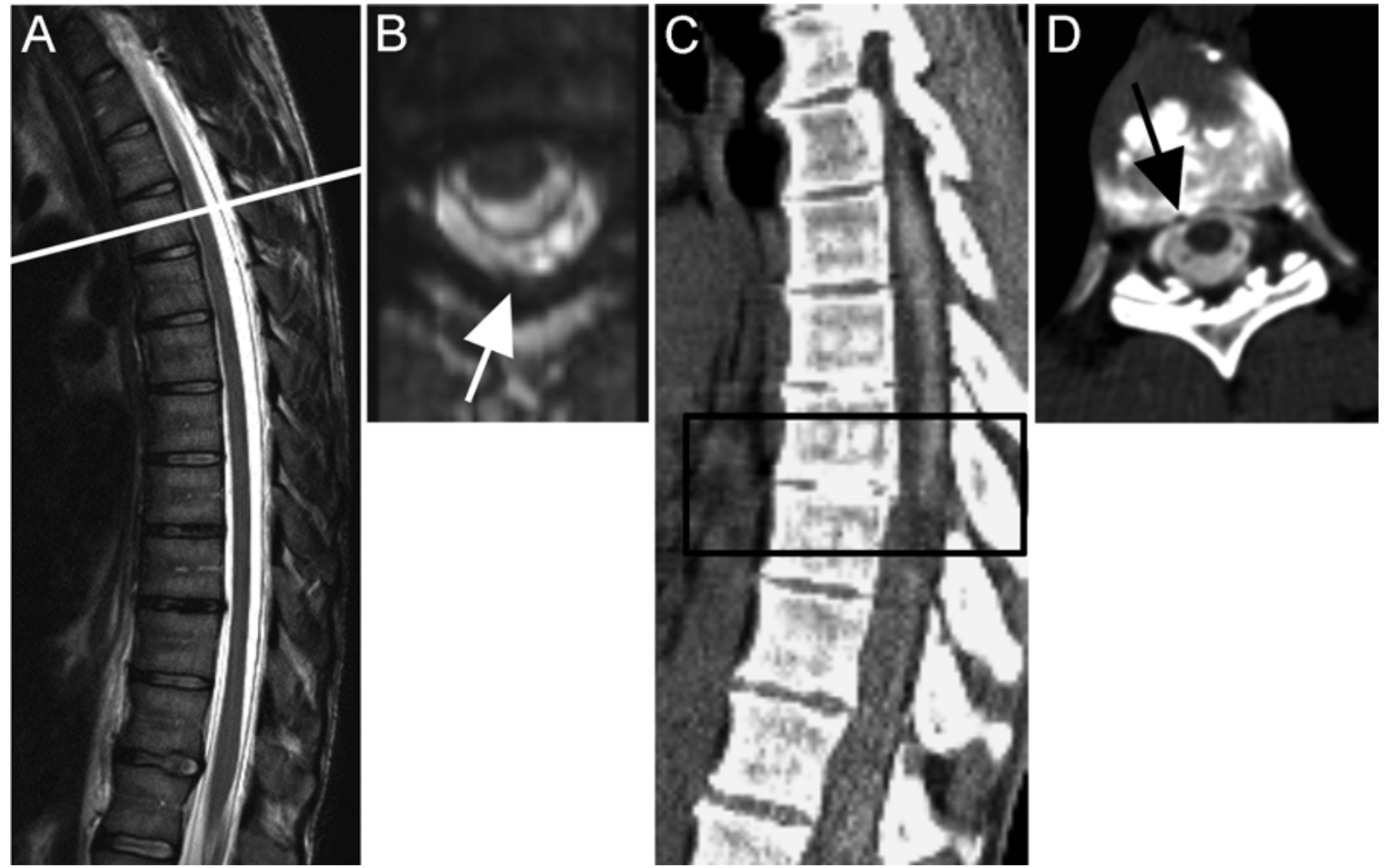

FIG. 2. A: Sagittal T2-weighted MR image showing increased extrathecal fluid collection spanning the lower cervical and entire thoracic spine. The line indicates the axial level in panel B. B: Axial T2-weighted CISS image showing the posterior thoracic extrathecal collection now measuring $5 \mathrm{~mm}$ in greatest thickness (arrow). C and D: Sagittal CT myelogram (C) showing a small disc osteophyte at T9-10 (box) and corresponding axial image at T9-10 (D) showing small ventral contrast extravasation (arrow).

\section{Postoperative Course}

At his 1-month postoperative follow-up visit, the patient reported complete resolution of headaches, being off of all pain medications, walking up to 1.5 hours without difficulty, and resuming work up to 7 hours at a time. Findings from examination remained normal at his baseline. He remains asymptomatic now 1 year after surgery.

\section{Discussion}

The incidence of symptomatic thoracic disc herniation is estimated to be 1 in 1,000,000, although pain and myelopathy rather than SIH are the more typical symptoms. ${ }^{5}$ Cases of SIH secondary to calcified thoracic discs are rare with little precedent as to optimal surgical intervention. This case illustrates the potential utility of the posterior laminectomy in nonmyelopathic patients without evidence of canal compromise and for whom neuromonitoring is available.

Historically, the first-line approach to SIH is medical treatment (keeping the patient supine and administering fluids, caffeine, and analgesia), ${ }^{19,21}$ followed by a secondline approach of an EBP. ${ }^{2,16,23}$ Surgical intervention is reserved for cases that are refractory to conservative management and EBP. Five of the 7 reported cases of thoracic disc-associated SIH in the literature were treated with an EBP alone. Of the cases requiring EBP, 1 had complete relief after $1 \mathrm{EBP},{ }^{1} 2$ required multiple $\mathrm{EBPs},{ }^{1,20}$ and 2 required both EBP and surgical evacuation of associated cranial subdural hematomas. ${ }^{1,27}$ Prior to this case report, only 2 other cases of surgically treated thoracic disc-associated SIH had been documented in the literature with
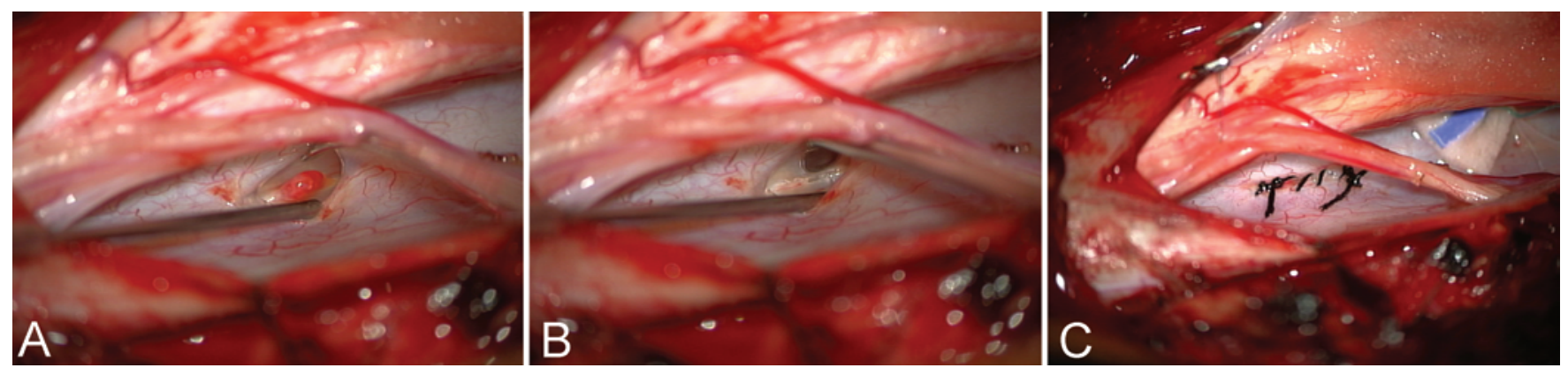

FIG. 3. Intraoperative images showing a small calcified disc through the ventral dural rent (A). After successful transdural discectomy the ventral dural defect is well delineated (B) and is closed primarily in a watertight fashion with running 5-0 silk suture (C). Figure is available in color online only. 
disparate methods of treatment. ${ }^{3,26}$ There is no standard or preferred approach for the surgical repair of spontaneous CSF leaks in the thoracic spine, and therefore we have drawn from the general literature with regard to the operative management of thoracic discs. Operative management of a similarly rare occurrence-SIH due to cervical intervertebral disc or osteophyte complex-has been reviewed previously. ${ }^{28}$ Given the unique cervical approaches used, namely, anterior cervical discectomy and fusion and anterior cervical corpectomy, we do not review the cervical spine literature here.

Surgical approaches to the thoracic spine consist of transthoracic (sternotomy, transpleural and retropleural), thoracoscopic, and posterolateral (costotransversectomy, lateral extracavitary, transpedicular pedicle sparing transfacet, and facetectomy) approaches. ${ }^{4,8,11,12,18,24}$ The two previously published cases of surgical treatment for SIH used standardly accepted thoracic techniques. Binder et al. reported on a patient with a ventral T2-3 disc osteophyte complex, which was repaired via a median sternotomy and anterior T2-3 discectomy with primary dural repair. ${ }^{3}$ Wilson et al. reported on a patient with T6-7 calcified disc-associated SIH refractory to 3 thoracolumbar EBPs, which ultimately required repair via a hemilaminectomy and costotransversectomy for disc excision without direct repair of the dura; indirect repair was performed using muscle, fat, fascia, and fibrin glue. ${ }^{26}$

Both the aforementioned anterior and posterolateral approaches can be cumbersome and associated with morbidity, particularly in young healthy patients. However, a less invasive, posterior laminectomy approach has been associated with poor outcomes, with literature dating back to the 1960s and, as a result, is widely discouraged. In recent years, this approach has been revisited., ${ }^{6,15}$ A group in South Korea reported their experience with the posterior approach for transdural discectomy in 3 patients with symptomatic, soft, paramedian discs located from T2 -3 to T11-12. In this case series, surgeons performed laminectomy without facetectomy with dorsal and ventral durotomies. Again, the iatrogenic ventral defect was not repaired primarily given the authors' impression that the ventral dura was adherent to the posterior longitudinal ligament, and the disc space was not extensively explored in an effort to limit the risk of injury to spinal cord. All discs that the authors attempted to treat were soft; calcified discs and disc osteophytes were excluded, and intraoperative neuromonitoring was not used. ${ }^{15}$ A second group in the Netherlands reported their experience with 13 transdural discectomies, including 4 calcified discs. Surgeons performed hemilaminectomy, partial facetectomy, and partial pediculectomy with posterolateral durotomy. As with the Korean group, the authors did not perform primary ventral dural closure, but rather used a synthetic dural patch and fibrin glue. One of 13 patients had a CSF leak. ${ }^{6}$

Here, we present our experience with the posterior laminectomy approach to a T9-10 calcified central disc herniation causing SIH and report for the first time successful treatment via posterior laminectomy and transdural discectomy with primary closure of both ventral and dorsal defects under direct visualization. Given our patient preference for conservative management with alacritous return to work, an anterior approach associated with potential morbidity would not have been consistent with the patient's interests. In light of this, we determined that the posterior approach was most appropriate for our patient, who did not have myelopathy or evidence of canal compromise and for whom neuromonitoring was available.

We acknowledge that this case is unique and recognize that there are limitations to broader applications of this approach. Of the numerous factors considered, our patient is a surgeon and could not comply with long-term activity limitations and sought a definitive repair after enduring long-standing symptoms. It is possible that in someone less active another EBP with more time to recover could have been beneficial. However, the presence of a small disc osteophyte favors surgical management. It is unlikely that the osteophyte complex would have resolved without intervention. In the only known study that followed asymptomatic thoracic discs with serial MR images, small disc herniations tended not to resolve but rather to remain the same or enlarge..$^{29}$ With threat of a persistent disc osteophyte, awaiting a spontaneous endogenous dural repair would be impractical. Additionally, this case report highlights the importance of preoperative imaging. The nearly radiographically occult ventral defect was best visualized preoperatively on the dynamic CT myelogram. In cases of CSF leak refractory to conservative management and with extraarachnoid CSF on MRI, dynamic CT myelography is the imaging modality of choice. ${ }^{10}$ Lastly, we acknowledge that despite the aforementioned case series touting the merit and safety of posterior thoracic laminectomy and transdural discectomy, this approach is still one that should be undertaken cautiously, if at all, and we recommend that all cases be performed by experienced surgeons with neuromonitoring and with the best interest of the patient in mind.

\section{Acknowledgment}

We thank K. Johnson for her assistance with preparation of the figures.

\section{References}

1. Allmendinger AM, Lee TC: Spontaneous intracranial hypotension from calcified thoracic disc protrusions causing CSF leak successfully treated with targeted epidural blood patch. Clin Imaging 37:756-761, 2013

2. Benzon HT, Nemickas R, Molloy RE, Ahmad S, Melen O, Cohen B: Lumbar and thoracic epidural blood injections to treat spontaneous intracranial hypotension. Anesthesiology 85:920-922, 1996

3. Binder DK, Sarkissian V, Dillon WP, Weinstein PR: Spontaneous intracranial hypotension associated with transdural thoracic osteophyte reversed by primary dural repair. Case report. J Neurosurg Spine 2:614-618, 2005

4. Bransford R, Zhang F, Bellabarba C, Konodi M, Chapman JR: Early experience treating thoracic disc herniations using a modified transfacet pedicle-sparing decompression and fusion. J Neurosurg Spine 12:221-231, 2010

5. Carson J, Gumpert J, Jefferson A: Diagnosis and treatment of thoracic intervertebral disc protrusions. J Neurol Neurosurg Psychiatry 34:68-77, 1971

6. Coppes MH, Bakker NA, Metzemaekers JD, Groen RJ: Posterior transdural discectomy: a new approach for the removal 
of a central thoracic disc herniation. Eur Spine J 21:623628, 2012

7. Fishman RA, Dillon WP: Dural enhancement and cerebral displacement secondary to intracranial hypotension. Neurology 43:609-611, 1993

8. Larson SJ, Holst RA, Hemmy DC, Sances A Jr: Lateral extracavitary approach to traumatic lesions of the thoracic and lumbar spine. J Neurosurg 45:628-637, 1976

9. Luetmer PH, Mokri B: Dynamic CT myelography: a technique for localizing high-flow spinal cerebrospinal fluid leaks. AJNR Am J Neuroradiol 24:1711-1714, 2003

10. Luetmer PH, Schwartz KM, Eckel LJ, Hunt CH, Carter RE, Diehn FE: When should I do dynamic CT myelography? Predicting fast spinal CSF leaks in patients with spontaneous intracranial hypotension. AJNR Am J Neuroradiol 33:690-694, 2012

11. Mack MJ, Regan JJ, Bobechko WP, Acuff TE: Application of thoracoscopy for diseases of the spine. Ann Thorac Surg 56:736-738, 1993

12. McCormick PC: Retropleural approach to the thoracic and thoracolumbar spine. Neurosurgery 37:908-914, 1995

13. Moayeri NN, Henson JW, Schaefer PW, Zervas NT: Spinal dural enhancement on magnetic resonance imaging associated with spontaneous intracranial hypotension. Report of three cases and review of the literature. J Neurosurg 88:912-918, 1998

14. Mokri B, Piepgras DG, Miller GM: Syndrome of orthostatic headaches and diffuse pachymeningeal gadolinium enhancement. Mayo Clin Proc 72:400-413, 1997

15. Moon SJ, Lee JK, Jang JW, Hur H, Lee JH, Kim SH: The transdural approach for thoracic disc herniations: a technical note. Eur Spine J 19:1206-1211, 2010

16. Nosik WA: Intracranial hypotension secondary to lumbar nerve sleeve tear. J Am Med Assoc 157:1110-1111, 1955

17. Paldino M, Mogilner AY, Tenner MS: Intracranial hypotension syndrome: a comprehensive review. Neurosurg Focus 15(6):ECP2, 2003

18. Patterson RH Jr, Arbit E: A surgical approach through the pedicle to protruded thoracic discs. J Neurosurg 48:768772,1978

19. Rando TA, Fishman RA: Spontaneous intracranial hypotension: report of two cases and review of the literature. Neurology 42:481-487, 1992

20. Rapport RL, Hillier D, Scearce T, Ferguson C: Spontaneous intracranial hypotension from intradural thoracic disc herniation. Case report. J Neurosurg 98 (3 Suppl):282-284, 2003

21. Schievink WI, Meyer FB, Atkinson JL, Mokri B: Spontane- ous spinal cerebrospinal fluid leaks and intracranial hypotension. J Neurosurg 84:598-605, 1996

22. Schievink WI, Morreale VM, Atkinson JL, Meyer FB, Piepgras DG, Ebersold MJ: Surgical treatment of spontaneous spinal cerebrospinal fluid leaks. J Neurosurg 88:243-246, 1998

23. Sencakova D, Mokri B, McClelland RL: The efficacy of epidural blood patch in spontaneous CSF leaks. Neurology 57:1921-1923, 2001

24. Sundaresan N, Shah J, Feghali JG: A transsternal approach to the upper thoracic vertebrae. Am J Surg 148:473-477, 1984

25. Vishteh AG, Schievink WI, Baskin JJ, Sonntag VKH: Cervical bone spur presenting with spontaneous intracranial hypotension. Case report. J Neurosurg 89:483-484, 1998

26. Wilson D, Christie J, Ferch R: Successful surgical treatment of intractable spontaneous intracranial hypotension due to a calcified thoracic disc prolapse. J Clin Neurosci 20:17731775,2013

27. Winter SC, Maartens NF, Anslow P, Teddy PJ: Spontaneous intracranial hypotension due to thoracic disc herniation. Case report. J Neurosurg 96 (3 Suppl):343-345, 2002

28. Witiw CD, Fallah A, Muller PJ, Ginsberg HJ: Surgical treatment of spontaneous intracranial hypotension secondary to degenerative cervical spine pathology: a case report and literature review. Eur Spine J 21 (Suppl 4):S422-S427, 2012

29. Wood KB, Blair JM, Aepple DM, Schendel MJ, Garvey TA, Gundry CR, et al: The natural history of asymptomatic thoracic disc herniations. Spine (Phila Pa 1976) 22:525-530, 1997

30. Yamamoto M, Suehiro T, Nakata H, Nishioka T, Itoh $H$, Nakamura T, et al: Primary low cerebrospinal fluid pressure syndrome associated with galactorrhea. Intern Med 32:228231,1993

\section{Author Contributions}

Conception and design: Borges. Analysis and interpretation of data: Pricola Fehnel. Drafting the article: Pricola Fehnel. Critically revising the article: both authors. Reviewed submitted version of manuscript: both authors. Approved the final version of the manuscript on behalf of both authors: Pricola Fehnel.

\section{Correspondence}

Katie Pricola Fehnel, Department of Neurosurgery, Massachusetts General Hospital, 55 Fruit St., Boston, MA 02114. email: kpricola@partners.org. 